J. Lake Sci. (湖泊科学), 2006, 18(6):627-635

http:// www.jlakes.org. E-mail: jlakes@ niglas.ac.cn.

(c) 2006 by Journal of Lake Sciences

\title{
太湖梅梁湾水体中介质吸收谱特性的空间变化
}

\author{
赵巧华 ${ }^{1,2,3}$, 张运林 ${ }^{1}$, 秦伯强 ${ }^{1 * *}$ \\ (1: 中国科学院南京地理与湖泊研究所,南京 210008) \\ (2: 中国科学院研究生院, 北京 100049) \\ (3: 南京信息工程大学环境科学与工程系, 南京 210044)
}

\begin{abstract}
摘 要: 水体中藻类颗粒物、非藻类颗粒物、CDOM 的光谱吸收特性的变化是整个水体对光吸收特性变化的源. 本文针对 梅梁湾水体中介质的吸收系数, 分析了总悬浮颗粒物、藻类颗粒物、非藻类颗粒物和 CDOM 的标准化谱吸收系数的变化. 结果表明: 藻类颗粒物、非藻类颗粒物组成比例的空间分布不同,是造成该区域总悬浮颗粒物标准化谱吸收系数空间变化 的原因,且标准化谱吸收系数的变化主要集中在 400-425 nm 及 $600-690 \mathrm{~nm}$ 两个波段; 就谱形而言,总悬浮颗粒物的谱 吸收特性主要表现为非藻类颗粒物的吸收特点,即在该区域的水体中,非藻类颗粒物是总悬浮颗粒物吸收特性的主要贡 献者; 藻类颗粒物、非藻类颗粒物和 CDOM 的吸收系数的空间变化主要是由各自的浓度变化造成的,即在采样时间内,梅 梁湾的水体中, 藻类颗粒物中的各种组成色素、非藻类颗粒物及 CDOM 中的腐殖酸和棕黄酸的组成比例在空间上基本没 有太大的变化. 另针对藻类颗粒物的吸收特性,本文还对比分析了用甲醇浸泡法和光谱标准分离法分离的藻类颗粒物的 吸收特性,发现相对光谱标准分离法来说,甲醇浸泡法会造成藻类颗粒物吸收系数的较大波动.
\end{abstract}

关键词：标准化吸收系数谱; 方差谱; 平均谱; 太湖

\section{Spatial variations of spectral absorption of medium in the Meiliang bay of Lake Taihu}

ZHAO Qiaohua ${ }^{1,2,3}$,ZHANG Yunlin ${ }^{1} \&$ QIN Boqiang ${ }^{1}$

(1:Nanjing Institute of Geography \& Limnology, Chinese Academy of Sciences, Nanjing 210008, P. R. China)

(2: Graduate School of the Chinese Academy of Sciences, Beijing 100039, P. R. China)

( 3 :Department of environment science and engineer, Nanjing university of information science and technology ,Nanjing 210044 ,P. R. China)

Abstract: The variations of the spectral absorption coefficient of natural water can be attributed to three principal factors-alga particles, nonalga particles and CDOM. Based on the absorption coefficients of the three factors in Meiliang bay, the variations in spectral absorption coefficient was analyzed. The result shows That the spatial variations of normalized spectral absorption coefficients of total particles are caused by the difference in alga particles:nonalga particles ratio, and the variance centralize between $400-425 \mathrm{~nm}$ and $600-690 \mathrm{~nm}$. As far as the spectral shape is concerned, there is prominent characteristic of nonalga particles in spectral absorption coefficients of total particles. Namely nonalga particles dominant the spectral absorption coefficients of total particles. The spatial variations in absorption coefficient of alga particles, nonalga, particles and CDOM are caused by the difference of their concentration. Namely, in the time of sampling, there is no evident difference in their constitutes of pigments for alga particles, and. humic and fulvic acid for nonalga particles and CDOM of the water in Meiliang bay. In addition, the absorption characteristic of alga particles separated by the method based on spectral criteria was analyzed and compared with that by methanol - extractions. We found that the absorption coefficients of latter exhibits significant fluctuation.

* 中国科学院知识创新战略行动项目 “长江中下游地区湖泊富养化发生机制与控制对策” KZCX1 - SW - 12 资助. 2005 - 12 - 30 收稿;2006-04-25 收修改稿. 赵巧华, 男,1972 生, 博士研究生, 讲师, E-mail: qhzhao@ niglas. ac. cn.

** 通讯作者:秦伯强, E-mail:qinbq@ niglas. ac. cn. 
Keywords : Normalized absorption; spectra variance apectra; mean spectra; Lake Taihu

水体吸收系数属于固有光学特性, 仅仅依赖于水体内介质本身的性质, 而独立于环境光场的变化(如 太阳光直射、漫射的分布、风引起的水表粗䊁程度对光场的改变、水底的反射吸收特性等 ${ }^{[1]}$. 在整个水生 生态系统中, 水体中各介质的吸收特性决定了它们对光的竞争能力, 从而决定了水体的初级生产力及水生 生态系统结构和功能的演化 ${ }^{[2]}$.

水体中的介质大致可以分为三大类, 其一是藻类颗粒物 (活体浮游植物). 它含有许多光合作用色 素 ${ }^{[1]}$, 在光学深度较大的水体中, 它是水生生态系统的主要生产者, 决定水体的初级生产力, 同时也是富营 养化水体中水华爆发的形成者. 其二是 CDOM, 它是一类含有腐殖酸和棕黄酸的可溶性有机物 ${ }^{[1-3]}$, 吸收的 能量与光化学反应有着密切的联系. 其三是非藻类颗粒物, 它是由浮游植物的残体、细菌、无机颗粒物等组 成的 ${ }^{[4]}$. 在二类水体中, 黄质和非藻类颗粒物的吸收占有重要的地位, 甚至是水体光学性质的决定因素, 其 吸收系数都呈 $\mathrm{e}$ 指数衰减趋势 ${ }^{[3]}$. 上述三类物质是水体中光的主要吸收者,共同决定水体吸收特性的变化. 而且它们三者之间存在对光的竞争关系，因而各介质的光谱吸收特性的变化决定了藻类光合作用的能力， 为了解浮游植物的种群、数量的变化及其与光场的关系提供了有力的工具; 在富营养化水体中, 浮游植物的 个别种群在有利的条件下会出现爆发性生长 ${ }^{[5]}$. 这种现象的形成机制也与藻类颗粒物的光谱吸收特性及 水体中有效光合辐射存在着密切的联系 ${ }^{[1]}$.

深人了解二类水体中各光学成分光谱吸收特性的变化规律, 有助于我们更深人地了解生物一光学模 型、离水辐射的信息和辐射在水中的传输规律 ${ }^{[1,3]}$.

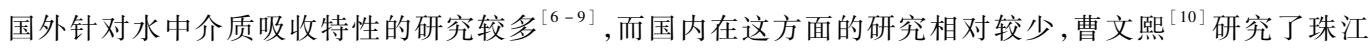
口悬浮颗粒物的吸收特性, 朱建华 ${ }^{[3]}$ 研究了黄海、东海非藻类颗粒物和 CDOM 的吸收光谱的斜率, 张运 林 ${ }^{[11]}$ 对太湖梅梁湾的藻类、非藻类颗粒物及 CDOM 的吸收系数的特征及其之间的关系作了一定的研究. 但 对介质的浓度和组成对谱吸收系数变化的贡献研究,却较为鲜见. 本文试图利用统计分析的方法, 揭示太湖 梅梁湾中的总悬浮颗粒物、藻类颗粒物、非藻类颗粒物和 CDOM 的组成变化的特点, 并分析造成谱吸收系 数变化的原因. 为研究该区域藻类颗粒物种群和数量的变化以及水质遥感提供依据.

\section{1 数据和方法}

\section{1 数据}

水样采自太湖梅梁湾的 16 个不同点 (图 1). 样品采集后放人冷藏箱内保存, 当天带回实验室进行过滤 处理, 然后放人冰箱内冷藏, 其中总悬浮颗粒物的吸收系数是利用定量滤膜技术 ${ }^{[12]}$ 在 $2-3 \mathrm{~d}$ 内获取的, 同 时测定了 CDOM 的吸收系数. 利用光谱标准法 ${ }^{[6]}$ 获得藻类颗粒物、非藻类颗粒物的吸收系数.

\section{2 理论与方法}

1.2.1 利用光谱标准法对悬浮颗粒物吸收系数进行分离 根据 Bricauad 和 Stramski ${ }^{[6]}$ 的研究, 通过浮游植 物在 $380 \mathrm{~nm}$ 与 $505 \mathrm{~nm}, 580 \mathrm{~nm}$ 与 $692.5 \mathrm{~nm}$ 的比例, 及非藻类颗粒物的吸收随波长呈 $e$ 的负指数衰减的特点, 建立方程组 (1)、(2).

$$
\begin{array}{r}
0.99 A \exp (-380 s)-A \exp (-505 s)=0.99 a_{p}(380)-a_{p}(505) \\
A \exp (-508 \mathrm{~s})-0.92 \mathrm{~A} \exp (-692.5 s)=a_{p}(580)-0.92 a_{p}(692.5)
\end{array}
$$

对于 $(1) 、(2)$ 两式, 可以通过二分法确定 $\mathrm{A} 、 \mathrm{~S}$, 然后根据文献 ${ }^{[6,14]}$ 计算出藻类颗粒物的吸收系数 $\left(a_{p h}(\lambda)\right)$ 和非藻类颗粒物的吸收系数 $\left(a_{d}(\lambda)\right)$.

1.2 .2 吸收光谱形状和振幅的分离 为了深人了解吸收光谱的变化, 需先引人光谱平均吸收系数 ${ }^{[4]}$ 的概 念, 其形式如下.

$$
a_{x}=\frac{\int a_{x}(\lambda) \mathrm{d} \lambda}{700-400}
$$

其中, $x$ 表示水体中的介质类型,当 $x$ 为 $\mathrm{t}$ 时,表示总悬浮颗粒物; 为 $\mathrm{ph}$ 时,表示藻类颗粒物; 为 $\mathrm{d}$ 时,表示非 藻类颗粒物; 为 $\mathrm{g}$ 时, 表示 CDOM. $a_{x}(\lambda)$ 为各类介质的谱吸收系数, $a_{x}$ 为光谱平均吸收系数. 


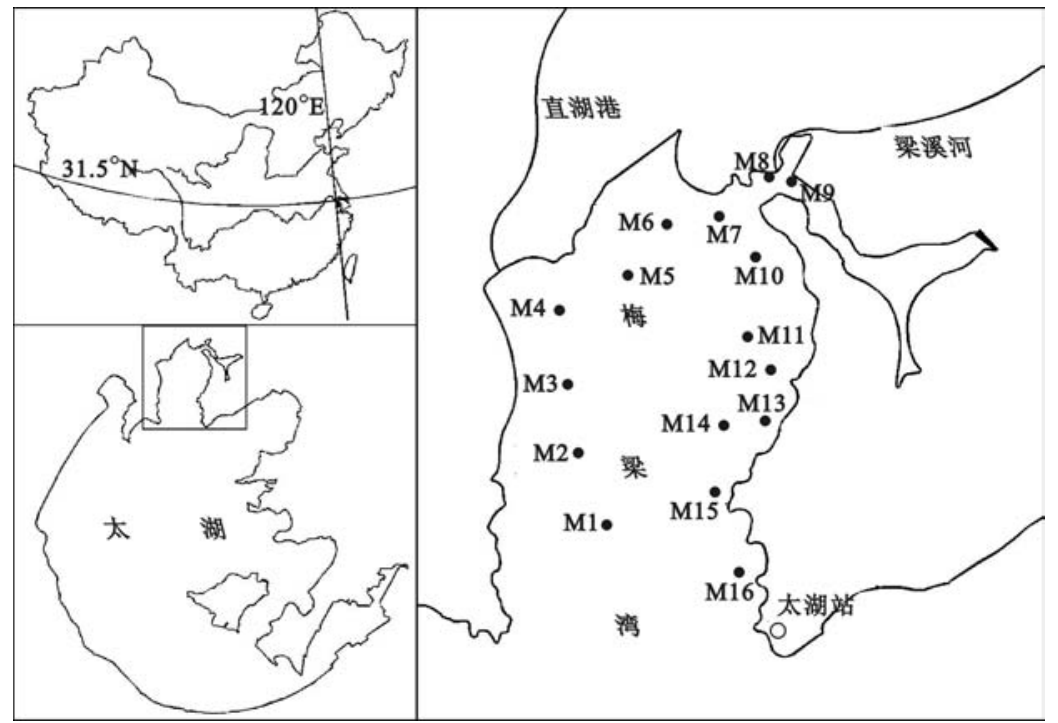

图 1 梅梁湾采样点位置

Fig. 1 Location of sampling stations in Meiliang Bay

水体中各介质吸收光谱展示了两个明显的变化. 其一是振幅的变化, 其中引起振幅变化的源也就是造 成光谱平均吸收系数变化的源 ${ }^{[4]}$, 即各介质中各成分的数量变化. 其二是光谱形状的变化. 它主要是由不 同色素在不同吸收波段的贡献形成的,即主要是色素的组成比例变化引起的 ${ }^{[4]}$, 当然, 就大颗粒而言. 其包 裹效应会导致吸收峰有所变平. 通过标准化谱吸收系数 $a_{x}^{n}(\lambda)$ 可以去除吸收系数中的浓度变化及大部分包 裹效应的影响,因而 $a_{x}^{n}(\lambda)$ 可以用来表征谱吸收系数的形状 ${ }^{[4]}$. 其形式如下:

$$
a_{x}^{n}(\lambda)_{k}=\frac{a_{x}(\lambda)_{k}}{a_{x k}}
$$

其中 $k$ 表示的是样本的标号.

由于标准化谱吸收系数 $a_{x}^{n}(\lambda)_{k}$ 表示的是第 $k$ 个样本, 光学成分为 $x$ 的光谱形状,而该形状又是由各自 相应的吸光成份组成比例决定的. 因而我们可以通过其数学期望和方差来探究标准化谱吸收系数发生较大 改变的波段, 从而分析其组成比例的变化, 尤其是在梅梁湾区域藻类颗粒物是否存在优势种群,为今后探究 藻类颗粒物种群的变化、初级生产力等提供有力的工具.

$$
\begin{gathered}
<a_{x}^{n}(\lambda)_{k}>=\frac{1}{N} \sum_{k=1}^{N} a_{x}^{n}(\lambda)_{k} \\
\sigma^{2}\left[a_{x}^{n}(\lambda)_{k}\right]=\frac{1}{N} \sum_{k=1}^{N}\left[a_{x}^{n}(\lambda)_{k}-<a_{x}^{n}(\lambda)_{k}>\right]^{2}
\end{gathered}
$$

其中, $\left\langle a_{x}^{n}(\lambda)_{k}>、 \sigma^{2}\left[a_{x}^{n}(\lambda)_{k}\right]\right.$ 分别是标准化谱吸收系数的平均谱和方差谱, $N$ 是样本数. 设 $A(\lambda)=<$ $a_{x}^{n}(\lambda)_{k}>$, 则 $a_{x}^{n}(\lambda)_{k}=A(\lambda) B_{K}(\lambda)$, 于是标准化谱吸收系数的方差谱可表示为:

$$
\sigma^{2}=\left[a_{x}^{n}(\lambda)_{k}\right]=A(\lambda)^{2}\left[\frac{1}{N} \sum_{k=1}^{N} B_{K}(\lambda)^{2}-1\right]
$$

从 (7) 式我们可以看出, $A(\lambda)^{2}$ 仍表示标准化谱吸收系数平均谱的形状,而 $\frac{1}{N} \sum_{k=1}^{N} B_{k}(\lambda)^{2}-1$ 的作用是 修正其方差谱的形状,但如果后者随变化不大时,则标准化谱吸收系数平均谱与其方差谱的形状是基本一 致的, 即可以说明各介质的吸光成分组成比例基本一致, 反之, 则可以通过 $\sigma^{2}\left[a_{x}^{n}(\lambda)_{k}\right]$ 来判断其组成比例 的变化. 


\section{2 分析与讨论}

\section{1 总悬浮颗粒物变化特征}

图 2 给出了总悬浮颗粒物标准化吸收谱,由于该谱刻画的是总悬浮颗粒物的谱形状,因而其反映的是 总悬浮颗粒物组成变化. 从图 2 中可以看出, 16 个采样点标准化吸收光谱大致可以分为两类, 其一, M 8 、 M9、M13 三个站点的谱在 $400-425 \mathrm{~nm}$ 范围内基本不随波长的减小而增大,而其余 13 个站点的谱却随波长 减小而增大. 根据 Mobley ${ }^{[1]}$ 、Kirk ${ }^{[2]}$ 、Prieur \& Sathyendranath ${ }^{[13]}$ 等的研究, 在 $400-425 \mathrm{~nm}$ 范围内, 藻类颗粒 物的吸收系数一般是随波长减小而减小,而非藻类颗粒物在 $400-700 \mathrm{~nm}$ 范围内均随波长减小而增大. 因 而在藻类颗粒物比例占一定优势的情况下,在 $400-425 \mathrm{~nm}$ 范围内, 总悬浮物颗粒物的吸收谱随波长减小 而减小, 反之, 则其吸收谱随波长减小而呈递增的趋势, 主要表现出非藻类颗粒物的吸收特征. 其二, 在 600 $-690 \mathrm{~nm}$ 范围内, 由于非藻类颗粒物的吸收系数衰减到红光区时相对较小,所以在该波段体现出较为明显 的藻类颗粒物的吸收特征, 主要表现出叶绿素 $\mathrm{a}$ 及其附属色素的吸收特征, 藻类颗粒物的比例越大, 则其峰 值在该波段体现得越明显. 从中可以得出 M8、M9、M13 三个站点的样本中藻类颗粒物相对其它点来说, 占 有一定的优势. 且根据藻类颗粒物所占的优势程度大小, 可将这三点排序为, 这与表 1 中的结果基本一致. 但这 16 个样本的总悬浮颗粒物标准化谱吸收系数基本呈非藻类颗粒物的特征, 表明在梅梁湾水体中, 悬浮 颗粒物的谱吸收系数主要体现的是非藻类颗粒物的吸收特性.

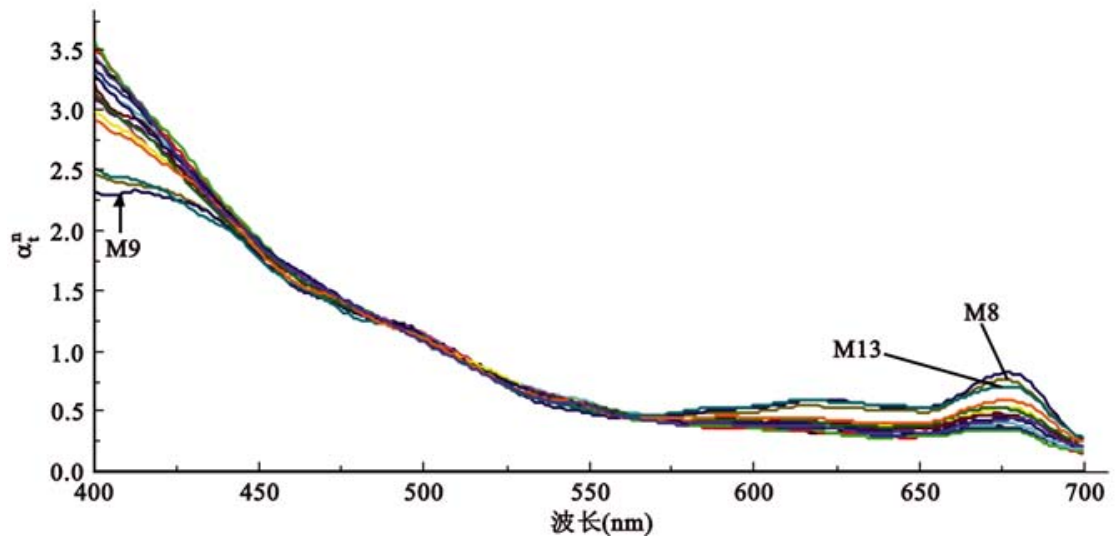

图 2 总悬浮颗粒物的标准化吸收谱

Fig. 2 the normalized absorption spectra of total particles

从图 1 给出的采样点位置分布来看, M8、M9 两点位于梁溪河河口,它们受梁溪河直接排放的影响最 大, 从而造成该两点藻类颗粒物浓度高,占总悬浮颗粒物中的比重大. 且由于在 M8 点附近有一小岛屿,使 得从河口到 M8 点的湖流变缓, 相对 M9 点而言, 其受直接输送的影响相对较小. 这与图 2 中 M8 点藻类颗粒 物的标准化吸收谱的特点及其与 M9 点的关系是基本一致的. 而 M13 点虽然比较接近梅梁湾口,但位于一 个小的湾口内, 使得其受湾外的影响较小,且离岸较近, 导致该点藻类颗粒物的比例较大,但较 M8、M9 点要 小. M4 点虽然位于直湖港港口, 但由于该港口近年一直处于关闭状态, 因而 M4 点并未表现出梁溪河河口 的特征.

表 1 叶绿素 $\mathrm{a}$ 的浓度

Tab. 1 The concentration of chlorophyll a

\begin{tabular}{ccccccccc}
\hline 点号 & M1 & M2 & M3 & M4 & M5 & M6 & M7 & M8 \\
\hline$\mu \mathrm{g} / \mathrm{L}$ & 41.5 & 39.3 & 40.9 & 45.7 & 64.1 & 118.5 & 65.3 & 128.6 \\
点号 & M9 & M10 & M11 & M12 & M13 & M14 & M15 & M16 \\
$\mu \mathrm{g} / \mathrm{L}$ & 180.2 & 94.2 & 73.7 & 65.9 & 123.3 & 56.8 & 65.3 & 42.1 \\
\hline
\end{tabular}



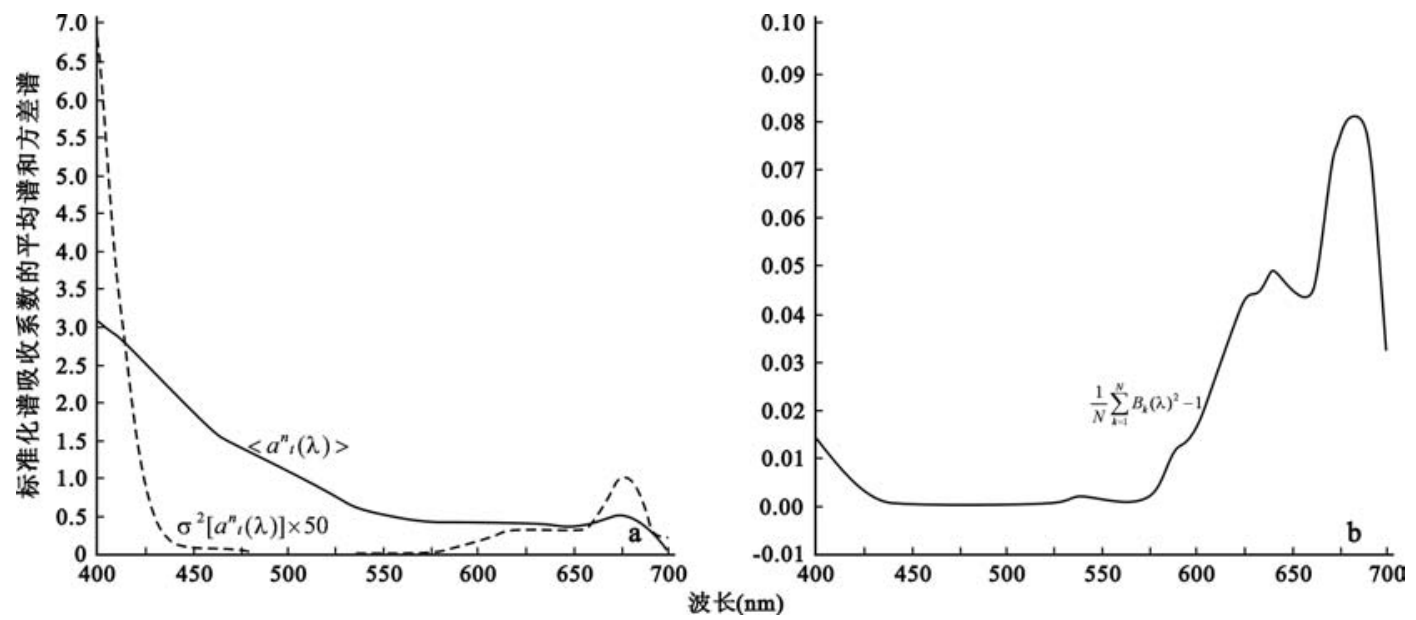

图 $3 \mathrm{a}$ : 总悬浮颗粒物的标准化谱吸收系数的平均谱 (实线) 和方差谱 (虚线)

$$
\mathrm{b}: \frac{1}{N} \sum_{k=1}^{N} B_{K}(\lambda)^{2}-1 \text { 随波长的变化( 总悬浮颗粒物) }
$$

Fig. 3 a: mean spectra (solid) and variance spectra (dashed) of normalized absorption coefficients of total particles;

$$
\text { b: variation of } \frac{1}{N} \sum_{k=1}^{N} B_{K}(\lambda)^{2}-1 \text { along with the wavelength (total particles) }
$$

从图 3b 可以看出, $\frac{1}{N} \sum_{k=1}^{N} B_{K}(\lambda)^{2}-1$ 随波长的变化主要体现在两个区域, 其一是在 $400-425 \mathrm{~nm}$ 波段中, 虽 然在该区域其变化不大,然而由于该波段值较大,因而在图 3a 中,其方差谱在此波段中表现出较大. 其二是 在 $600-690 \mathrm{~nm}$ 区域内, 在该区域有一主峰在 $675 \mathrm{~nm}$ 左右; 有一肩峰是在 $640 \mathrm{~nm}$ 左右. 虽然此两处的峰值 较大,然而由于相对较小,所以在图 3a 中其方差谱在该区域内有所体现,但却不如 $400-425 \mathrm{~nm}$ 范围内 突出.

在图 $3 \mathrm{~b}$ 中,曲线随波长的起伏较大, 说明总悬浮颗粒物的组成有着较大的区别. 在 $400-425 \mathrm{~nm}$ 和 600 $-690 \mathrm{~nm}$ 两个区域内,变化主要是由藻类颗粒物中的叶绿素 a 与非藻类颗粒物的吸收特性造成的. 因而可 以说在这 16 个样本中, 其藻类和非藻类颗粒物的组成比例变化较大; 对梅梁湾总颗粒物的谱吸收系数来 说,其变化是由藻类颗粒物、非藻类颗粒物组成比例的变化造成的,且标准化谱吸收系数的变化主要集中在 $400-425$ 和 $600-690$ 两个波段内. 由此可见, $\frac{1}{N} \sum_{k=1}^{N} B_{K}(\lambda)^{2}-1$ 能更好地刻画标准化谱吸收系数变化的波 带位置.

\section{2 藻类颗粒物的变化特征}

2.2 .1 基于光谱标准法分离的藻类颗粒物的变化特征 从图 4 中可以看出, 16 个站点藻类颗粒物的标准化 吸收谱的形状基本一致,体现了活体藻类的主要吸收特征,然而在其组成上是否存在变化,还应从标准化吸 收系数的方差谱和平均谱中寻求.

在图 5b 中,存在三个主要的峰值,其一是在 400-425 nm 左右. 由于该区域对应的标准化吸收谱值相 应较大,因而其方差谱在相应的波段也体现得非常明显,然而该处的变化体现的不是藻类颗粒物的吸收特 征,而应是非藻类颗粒物的吸收特征. 其二是在 $565 \mathrm{~nm}$ 左右,但由于藻类颗粒物在该波段的吸收系数较小 (图 5a), 导致其在方差谱中的出现并不特别突出. 其三是在 $690-700 \mathrm{~nm}$, 藻类颗粒物在该区域的吸收系数 也是非常小的,因而其在方差谱中体现的不明显. 总的来说, 在图 $5 \mathrm{~b}$ 中的三个峰值均是在藻类颗粒物变化 最小的区域出现, 因而可以判断其体现的并不是藻类颗粒物吸收系数的变化, 就其原因可能是测量时数据 的波动和用光谱标准法分离时造成的误差引起的. 剔除上述三个峰值区, 藻类颗粒物的 $\frac{1}{N} \sum_{k=1}^{N} B_{K}(\lambda)^{2}-1$ 变 


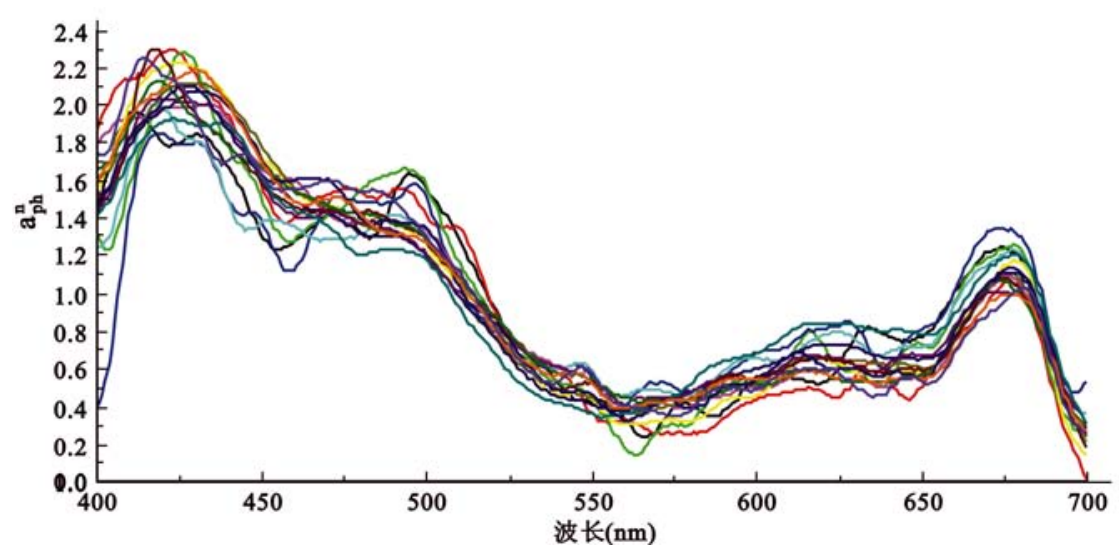

图 4 藻类颗粒物 (用光谱标准法分离) 的标准化吸收谱

Fig. 4 the normalized absorption spectra of alga particles (separated based on spectral criteria)
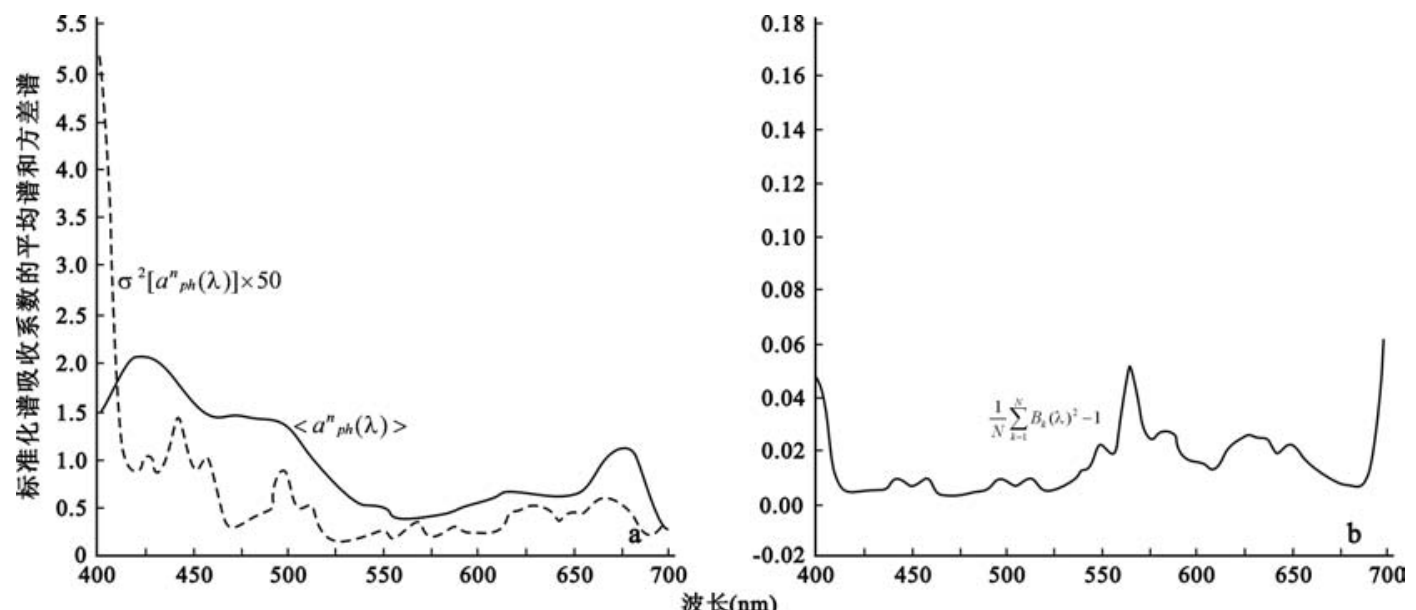

图 $5 \mathrm{a}$ : 藻类颗粒物的标准化谱吸收系数的平均谱 (实线) 和方差谱 (虚线) (光谱标准法分离) $\mathrm{b}: \frac{1}{N} \sum_{k=1}^{N} B_{K}(\lambda)^{2}-1$ 随波长的变化(光谱标准法分离的藻类颗粒物)

Fig. 5 a:mean spectra (solid) and variance spectra (dashed) of normalized absorption coefficients of alga particles ( separated based on spectral criteria)

b: variation of $\frac{1}{N} \sum_{k=1}^{N} B_{K}(\lambda)^{2}-1$ along with the wavelength (alga particles separated based on spectral criteria) 化较小, 且没有较大的起伏, 尤其是在其吸收系数较大的区域 (图 5a). 因而其基本成一直线状, 可以粗略地 说它基本上是独立于的,也就是说藻类颗粒物中的组成色素比例在该区域内没有太大的变化,即在梅梁湾 区内, 藻类种群变化相对不大, 这与夏季梅梁湾蓝藻爆发, 优势种明显 ${ }^{[15]}$ 的事实也是吻合的. 同时从其方差 谱和平均谱的形状来看, 它们的形状基本是一致的, 这也从侧面证实了上述的分析.

2.2 .2 基于甲醇提取法分离的藻类颗粒物的变化特征 图 6 中描述的是用甲醇提取法分离的藻类颗粒物 的标准化吸收谱, 在 400-425 nm 区域,一部分样本的标准化吸收谱是随波长减小而增大的, 其明显体现了 非藻类颗粒物的吸收特征, 这说明对于非藻类颗粒物来源广泛、浓度较高的水体来说, 用甲醇提取法分离藻 类颗粒物会将非藻类颗粒物中的非光合作用色素一并提取,系统放大藻类颗粒物的吸收系数. 


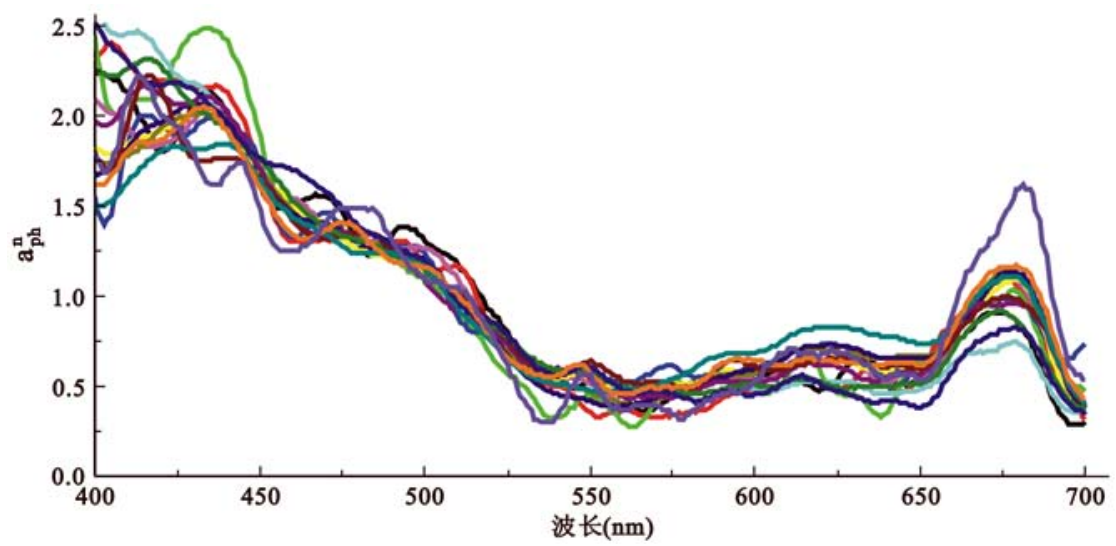

图 6 藻类颗粒物的标准化吸收系数谱 (甲醇法分离)

Fig. 6 Normalized absorption spectra of alga particles (separated based on methanol-extractions)
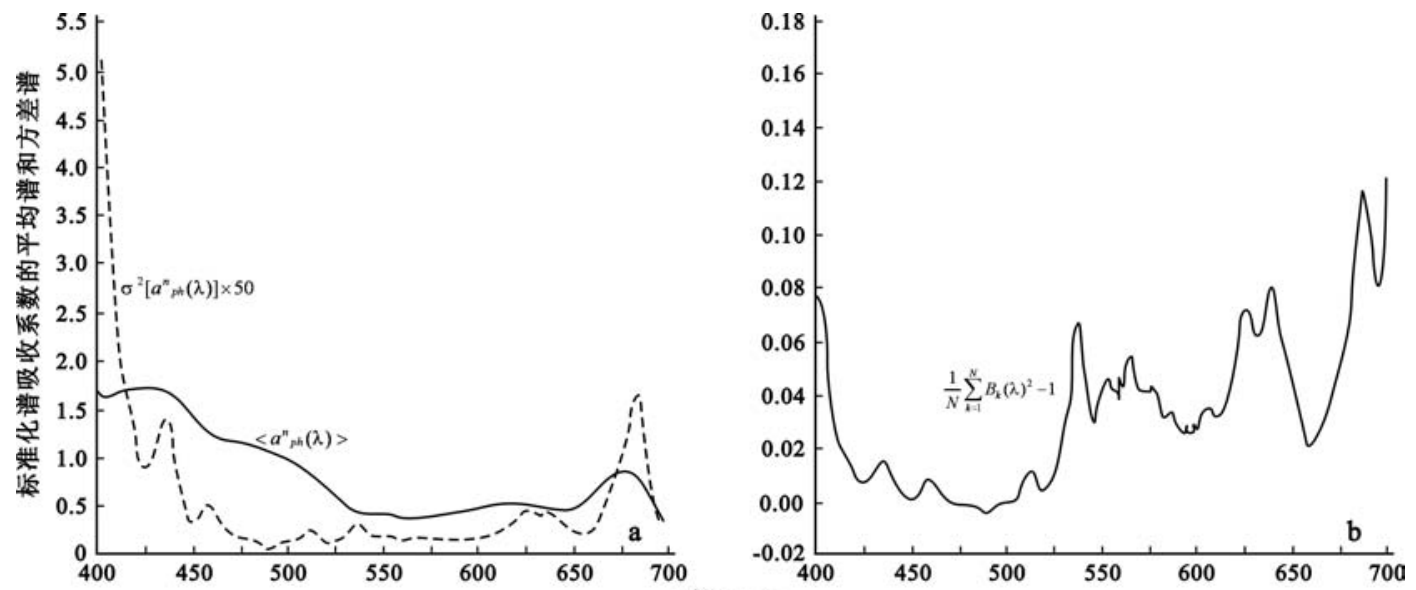

图 $7 \mathrm{a}$ : 藻类颗粒物的标准化谱吸收系数的平均谱 (实线) 和方差谱 (虚线) (甲醇提取法分离) $\mathrm{b}: \frac{1}{N} \sum_{k=1}^{N} B_{K}(\lambda)^{2}-1$ 随波长的变化(甲醇提取法分离的藻类颗粒物)

Fig. 7 a:mean spectra (solid) and variance spectra (dashed) of normalized absorption coefficients of alga particles. ( separated based on methanol-extractions) b: variation of $\frac{1}{N} \sum_{k=1}^{N} B_{K}(\lambda)^{2}-1$ along with the wavelength

( alga particles separated based on methanol-extractions)

在图 7b 中, $\frac{1}{N} \sum_{k=1}^{N} B_{K}(\lambda)^{2}-1$ 除了表现出与图 $5 \mathrm{~b}$ 中的峰值以外, 而且还存在其他几个的峰值, 且该曲 线随波长的变化相对比较剧烈, 但整体的变化范围并不是特别大, 因而该曲线也能得出藻类颗粒物中组成 色素的比例变化不大的结论, 只是其趋势不如用光谱标准法分离法所得的结果明显. 其主要原因是用甲醇 提取了的脱镁色素引起的干扰. 这在图 7a 中的 $630 、 680 \mathrm{~nm}$ 左右的峰值体现得较为明显.

\section{3 非藻类颗粒物及 CDOM 的变化特征}

图 8a 描述的是非藻类颗粒物标准化吸收系数的平均谱和方差谱,其方差谱最大的区域是在 $400-425$ 

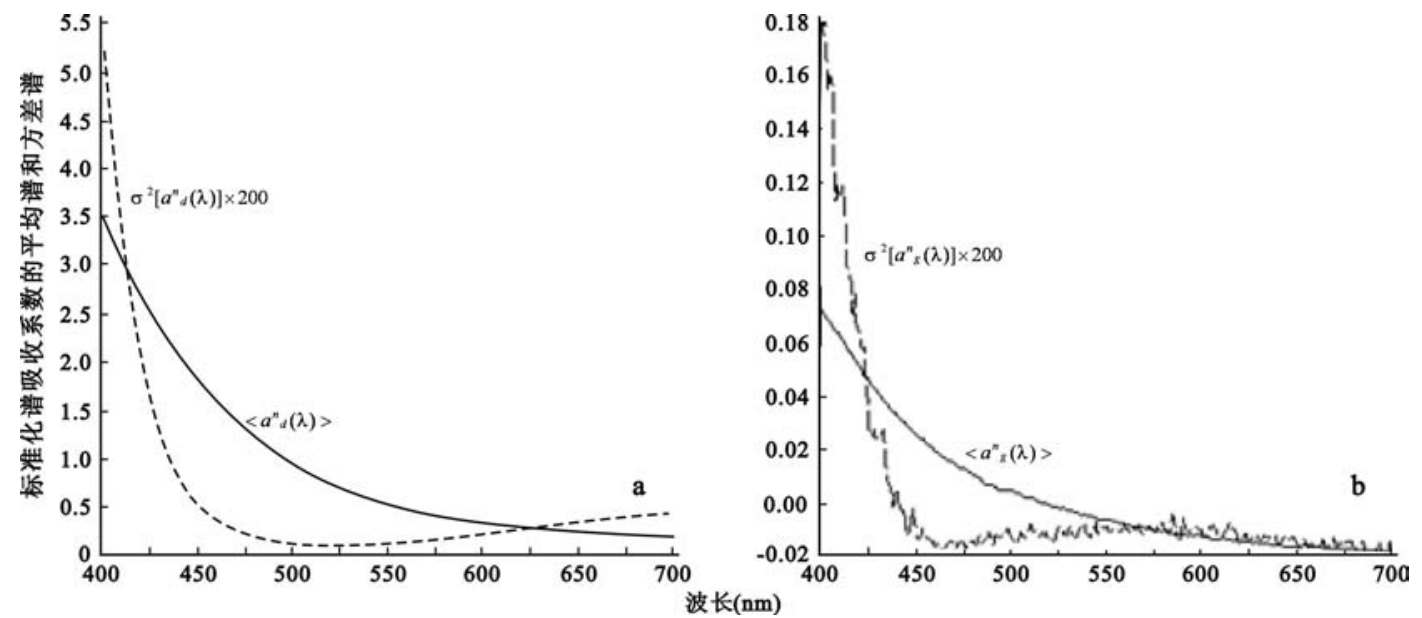

图 $8 \mathrm{a}$ : 非藻类颗粒物的标准化谱吸收系数的平均谱(实线) 和方差谱 (虚线); $\mathrm{b}:$ CDOM 的是标准化谱吸收系数的平均谱 (实线) 和方差谱 (虚线)

Fig. 8 a: mean spectra (solid) and variance spectra (dashed) of normalized absorption coefficients of nonalga particles;

b: mean spectra (solid) and variance spectra (dashed) of normalized absorption coefficients of CDOM

$\mathrm{nm}$ 波段,这主要体现出其组成上有一定的变化, 即腐殖酸和棕黄酸比例有一定的变化,其它区域,其方差谱 变化不大. 然而由于我们对该方差谱放大了 200 倍, 所以尽管在远蓝光区的方差有峰值, 但实际上其值非常 小, 因而可以说在该研究区域非藻类颗粒物中的腐殖酸和棕黄酸的组成比例在空间上没有太大的变化. 在 图 $8 \mathrm{~b}$ 中可以得到类似的结论, 即 CDOM 中的腐殖酸和棕黄酸的组成比例在空间上没有太大的变化;马荣华 等 ${ }^{[16]}$ 的研究认为, 在不同湖区, 同一湖区的不同季节 CDOM 的吸收光谱的影响参数可能不同, 然而本文研 究的是同一时间的梅梁湾区域,因而,上述结论也基本与其研究结果较为吻合.

\section{3 结论}

(1) 在梅梁湾中, 藻类颗粒物、非藻类颗粒物组成比例的空间分布不同, 是造成该区域总悬浮颗粒物标 准化谱吸收系数空间变化的原因,且标准化谱吸收系数的变化主要集中在 $400-425 \mathrm{~nm}$ 及 $600-690 \mathrm{~nm}$ 两 个波段.

(2) 就悬浮颗粒物的谱形而言,梅梁湾中的谱吸收特性主要体现出非藻类颗粒物的吸收特性, 说明在 该区域水体中,非藻类颗粒物是总悬浮颗粒物吸收特性的主要贡献者.

(3) 藻类颗粒物、非藻类颗粒物和 CDOM 的吸收系数的空间变化主要是由其各自的浓度变化造成的, 即在采样时间内, 梅梁湾的水体中, 藻类颗粒物中的各种组成色素、非藻类颗粒物及 CDOM 中的腐殖酸和 棕黄酸的组成比例在空间上基本没有太大的变化.

(4) 相对光谱标准分离法来说, 甲醇浸泡法会造成藻类颗粒物吸收系数的较大波动.

\section{4 参考文献}

[1] Mobley C D. Light and Water Radiative Transfer in Nature Waters. New York: Academic Press,1994:60.

[2] Kirk J T O. Light and photosynthesis in aquatic ecosystems. Cambridge University Press, 1994:Xi .

[3] 朱建华,李桐基. 黄东海非色素颗粒与黄色物质的吸收系数光谱模型研究,海洋技术, 2004,23 (2):7 -13 .

[4] Roesler C S. and Perry M J. Modeling in situ phytoplankton absorption from total absorption spectra in productive inland marine waters. Limnol \& Oceanogr, 1989,34(8):1510-1523.

[ 5] 秦伯强, 高 光, 胡维平等. 浅水湖泊生态系统恢复的理论与实践的思考. 湖泊科学, 2005,17(1):9- 
16 .

[6] Bricaud, A \& Stramski D. Spectral absorption coefficients of living phytoplankton and nonalgal biogenous matter: A comparison between the Peru upwelling and the Sargasso Sea. Limnol \& Oceanogr, 1990, 35 (3) : $562-582$.

[7] Lutz V A, Sathyendranath S \& Head E J H. Absorption coefficient of phytoplankton: regional variations in the North Atlantic. Marine Ecology Progress Series, 1996, 135: 197 - 213.

[8] Babin M, Stramsk D, Ferrar G M, et al. Variations in the light absorption coefficients of phytoplankton, nonalgal particles, and dissolved organic matter in coastal waters around Europe. J Geophys Res, 2003, 108: $1-20$.

[9] Sasaki H, Miyamura T, Saitoh S I, et al. Seasonal variation of absorption by particles and colored dissolved organic matter (CDOM) in Funka Bay, southerwestern Hokkaido, Japan. Estuar Coas Shelf S, 2005,64 : $447-458$.

[10] 曹文熙,杨跃中, 许晓强等. 珠江口悬浮颗粒物的吸收光谱及其区域模式. 科学通报, 2003,48(17): $1876-1882$.

[11] 张运林,秦伯强, 杨龙元. 太湖梅梁湾水体悬浮颗粒物和 CDOM 的吸收特性. 生态学报,待刊.

[12] Cleveland J S \& Weidemann A D. Quantifying absorption by aquatic particles: A multiple scattering correction for glass-fiber filters. Limnol \& Oceanogr, 1993, 38(6): $1321-1327$.

[13] Louis Prieur and Shubha Sathyendranath. An optical clasification of coastal and oceanic waters based on the specific spectrsl absorption curves of phytoplankton pigments, dissolved organic matter, and other particulate materials. Limnol \& Oceanogr, 1981,26(4): $671-689$.

[14] 赵巧华,张运林, 秦伯强. 太湖梅梁湾水体悬浮颗粒物吸收系数的分离. 湖泊科学, 2006,18 (4):356 -362 .

[15] 陈宇炜,高锡云,秦伯强. 西太湖北部夏季藻类种间关系的初步研究. 湖泊科学, 1998,10(4):35-40.

[16] 马荣华,戴锦芳, 张云林. 东太湖 CDOM 吸收光谱的影响因素与参数确定. 湖泊科学, 2005,17(2):120 -126 . 